\title{
Coupled Vibration of Cracked Frame with Damping
}

\author{
M. BOLD* AND W. SOCHACKI \\ Częstochowa University of Technology, 42-201 Częstochowa, Poland \\ Doi: $10.12693 /$ APhysPolA.138.236 \\ *e-mail: bold@imipkm.pcz.pl
}

\begin{abstract}
This paper presents an analytical study of vibrations of the cracked discrete-continuous system with viscous damping. The influence of viscous external, internal and structural damping on axial and lateral vibrations of cracked frame was investigated. The adopted model was Bernoulli-Euler beam model where the virtual work of non-conservative forces comes from external, internal and constructional damping of supports. The obtained results allow to determine some geometrical parameters at which the amplitude decay factor is the highest. The parameters identified on the basis of study are the depth and location of the crack, and the range of values of the constructional damping coefficient.
\end{abstract}

topics: damping, portal frame, open crack, amplitude decay factor

\section{Introduction}

Cracks are structural defects that modify the dynamic behaviour of the construction and can reduce the efficiency of the system/machine mainly due to their influence on object vibrations. A mathematical model of real systems which takes into account the possible occurrence of cracks and the different source of energy dissipation (damping phenomenon), helps to determinate the dynamic properties of real systems and identification of its damage.

The dynamic analysis of frame structures was presented in [1-5]. The study of [1] and [2] concerned on constructional damped vibration of $\Gamma$-frame and impact of complex damping on vibration amplitude of the portal frame, respectively. The formulation and solution of the problem of transverse damped vibrations of $\Gamma$-frame with crack was presented in [3]. In that paper, the influence of crack localization and values of constructional and internal damping on complex eigenvalue of the frame were examined. The noninvasive method of cracks identification is proposed in [4]. The method based on the transverse open single-sided crack which is modelled using rotational spring (calculated stiffness) and the force displacement relationship is described by local flexibility matrix. The problem of instability three members slender system with crack in the internal rod based of a static criterion of instability was presented in work [5]. Analysed system composed of several elements was subjected to Euler's load, while the internal crack size was reflected by rotational spring.

Author's previous studies concerned the analysis of various frame shapes with one kind of damping, selected vibrations or single cracked structure. The influence of material and external damping on vibrations of frame with and without damage is still the field to research. The present paper examined the axial and lateral vibrations of frame with 2 one-side cracks by considering the influence of viscous complex damping on system. The range of values of the constructional damping coefficient, as well as the depth and location of cracks, are the parameters identified on the basis of this study. It turns out, that the various values of damping coefficients, cracks depth and cracks localization change quantitative and qualitative the amplitude of decay factor and damped frequency.

\section{Boundary problem}

To formulated the boundary problem of axial and lateral vibrations of the system, we used the Bernoulli-Euler beam model where the virtual work of non-conservative forces come from complex damping: external damping of medium surrounding the system, internal damping of viscoelastic material of the model, and constructional damping of supports. In the adopted model (see Fig. 1) the stiffness of spring $K_{m}$ was used to model the crack. In the local flexibility matrix, the material compliance of crack was generated by the rotational rigidity using the form as in [4]. Namely

$$
K_{m}=\frac{1}{c_{m}},
$$

where

$$
c_{m}=5.346 \frac{h}{E_{n} J_{n}} I_{m}\left(\frac{a_{m}}{h}\right),
$$




$$
\begin{aligned}
& I_{m}\left(\frac{a_{m}}{h}\right)=1.8624\left(\frac{a_{m}}{h}\right)^{2}-3.95\left(\frac{a_{m}}{h}\right)^{3} \\
& +16.375\left(\frac{a_{m}}{h}\right)^{4}-37.226\left(\frac{a_{m}}{h}\right)^{5} \\
& +76.81\left(\frac{a_{m}}{h}\right)^{6}-126.9\left(\frac{a_{m}}{h}\right)^{7}+172\left(\frac{a_{m}}{h}\right)^{8} \\
& -143.97\left(\frac{a_{m}}{h}\right)^{9}+66.56\left(\frac{a_{m}}{h}\right)^{10} .
\end{aligned}
$$

In the above formulas one has compliance $c_{m}$, dimensionless local compliance function $I_{m}$ [4], a crack depth $a_{m}$, and a beam height $h$, where $m=1,2$.

The boundary problem was formulated on the basis on Hamilton's principle, i.e.,

$$
\delta \int_{t_{1}}^{t_{2}}(T-V) \mathrm{d} t+\int_{t_{1}}^{t_{2}} \delta W_{N} \mathrm{~d} t=0 .
$$

In turn, kinetic $(T)$ and potential $(V)$ energies, and virtual work of non-conservative forces $\left(\delta W_{N}\right)$ were expressed as:

$$
T=\frac{1}{2} \sum_{n=1}^{5} \frac{\rho_{n} F_{n}}{2} \int_{0}^{l_{n}}\left(\frac{\partial W_{n}\left(x_{n}, t\right)}{\partial t}\right)^{2} \mathrm{~d} x_{n},
$$

It should be read that $W_{n}(x, t)$ - transverse displacements, $U_{n}(x, t)$ - longitudinal displacements, $E_{n}$ - Young's modulus, $E_{n}^{*}$ - material viscosity, $F_{n}-$ cross-sections, $J_{n}-$ moment of inertia in frame cross-sections, $l_{n}$ - length of the beam, $L_{C}$ - total length of frame, $C_{R}$ - constructional viscous damping, $C_{E}$ - external viscous damping, $\rho_{n}-$ material densities, $x_{n}-$ spatial coordinate, $t$ - time, and $n=1,2, \ldots 5$.

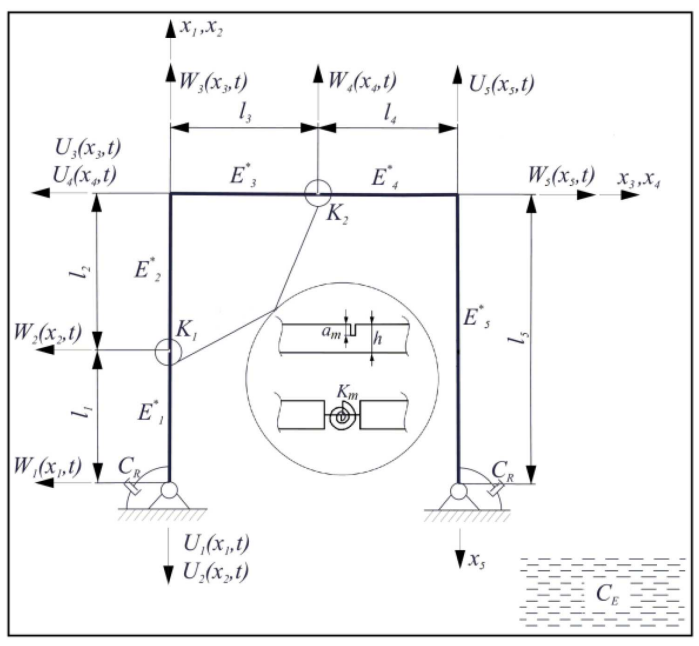

Fig. 1. Scheme of the examined system.
The differential equations of motion in the axial (8) and lateral direction (9) had the following form:

$$
\begin{aligned}
& J_{n} E_{n} \frac{\partial^{4} W_{n}\left(x_{n}, t\right)}{\partial x_{n}^{4}}+J_{n}\left(E_{n}+E_{n}^{*}\right) \frac{\partial^{5} W_{n}\left(x_{n}, t\right)}{\partial x_{n}^{4} \partial t} \\
& +C_{E} \frac{\partial W_{n}\left(x_{n}, t\right)}{\partial t}+\rho_{n} F_{n} \frac{\partial^{2} W_{n}\left(x_{n}, t\right)}{\partial t^{2}}=0,(8) \\
& -F_{n} E_{n} \frac{\partial^{2} U_{n}\left(x_{n}, t\right)}{\partial x_{n}^{2}}-F_{n}\left(E_{n}+E_{n}^{*}\right) \frac{\partial^{3} U_{n}\left(x_{n}, t\right)}{\partial x_{n}^{2} \partial t} \\
& +\rho_{n} F_{n} \frac{\partial^{2} U_{n}\left(x_{n}, t\right)}{\partial t^{2}}=0 .
\end{aligned}
$$

The boundary problem of the free vibrations of the considered non-conservative (due to damping) system was solved numerically for the eigenvalues $\omega^{*}$. The complex numbers $\omega^{*}$ represent the damped vibration frequencies $\operatorname{Re}\left(\omega^{*}\right)$ and amplitude decay factor $\operatorname{Im}\left(\omega^{*}\right)$, i.e.,

$$
\omega^{*}=\operatorname{Re}\left(\omega^{*}\right) \pm i \operatorname{Im}\left(\omega^{*}\right) \text {. }
$$

The dimensionless parameters introduced in the calculation were

$$
\begin{aligned}
& \eta=\frac{E_{n}^{*}}{h E_{n}}, \quad \nu=\frac{C_{e} L_{C}^{3}}{d}, \\
& \mu=\frac{c_{R}}{d}, \quad L=\frac{\left(l_{1}+l_{2}\right)}{l_{3}},
\end{aligned}
$$


where

$$
\begin{aligned}
& L_{C}=l_{1}+l_{2}+l_{3}+l_{4}+l_{5}, \\
& h^{2}=\frac{\rho_{n} A_{n}}{E_{n} J_{n}} L_{C}^{4}, \quad d^{2}=L_{C}^{2}\left(\rho_{n} A_{n} E_{n} J_{n}\right) .
\end{aligned}
$$

Geometrical and material data were set accordingly: lengths $l_{1}=1-4 \mathrm{~m}, l_{2}=2 \mathrm{~m}, l_{3}=1-4 \mathrm{~m}$, diameter of beams $D_{n}=0.05 \mathrm{~m}$, Young modulus $E_{n}=2.1 \times 10^{11} \mathrm{~Pa}$, external damping coefficient $\nu=0.49 \times 10^{-6}\left(\right.$ or $C_{E}=1.0041 \times 10^{-3} \mathrm{~N} \mathrm{~s} / \mathrm{m}^{2}$ of still fresh water $[6,7])$, internal damping coefficient $\eta=0-2$ (for homogeneous structural steel [6-8]), constructional damping coefficient $\mu=0-2$, material density $\rho_{n}=7.7 \times 10^{3} \mathrm{~kg} / \mathrm{m}^{3}$.

\section{Results}

The calculations results of this paper are presented in Figs. 2-7. The relationships of the real $\operatorname{Re}\left(\omega^{*}\right)$ and the imaginary $\operatorname{Im}\left(\omega^{*}\right)$ parts of the first eigenvalue of the frame, for different locations of first $L_{1}$ and second $L_{2}$ crack together with their depth $a_{1} / h=a_{2} / h=a / h$, are shown in Figs. 2 and 3 , respectively. The values of damping coefficients are $\eta=0.002, \nu=10^{-5}$ and $\mu=1$.

The propagation of functions described the damped frequency and amplitude decay factor of first eigenvalue with simultaneous change of the first crack depth and crack location, for constant values of $a_{2} / h$ and $L_{2}$ are presented in Fig. 4. Analogical dependencies for second crack are presented in Fig. 5. The values of damping coefficients were $\eta=0.002, \nu=10^{-5}$ and $\mu=1$. Substantial changes can be observed especially in $\operatorname{Re}\left(\omega^{*}\right)$ and $\operatorname{Im}\left(\omega^{*}\right)$ for different location and depth of the first crack. In both Figs. 4 and 5 one can also observe the areas in the progression of curves which are non-monotonic.

Figures 6 and 7 present the dependence of the real and the imaginary part of the first eigenvalue of frame on changes in constructional damping coefficient $\mu$. The values of coefficients are $\nu=10^{-5}$, $\eta=\{0,0.002,00.4\}$ and selected values of cracks depth and location. Main changes can be observed in value of damped frequency and amplitude decay factor of the system with changes the value of constructional damping $\mu$.

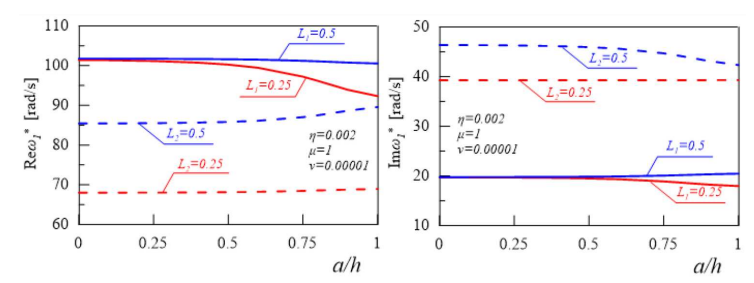

Fig. 2. The relationship between $\operatorname{Re}\left(\omega^{*}\right)$ and $\operatorname{Im}\left(\omega^{*}\right)$ of the first eigenvalue of the frame and cracks depth $a / h$.
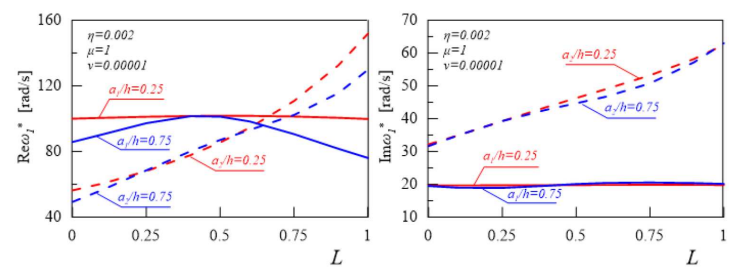

Fig. 3. The relationship between $\operatorname{Re}\left(\omega^{*}\right)$ and $\operatorname{Im}\left(\omega^{*}\right)$ of the first eigenvalue of the frame and cracks location $L$.

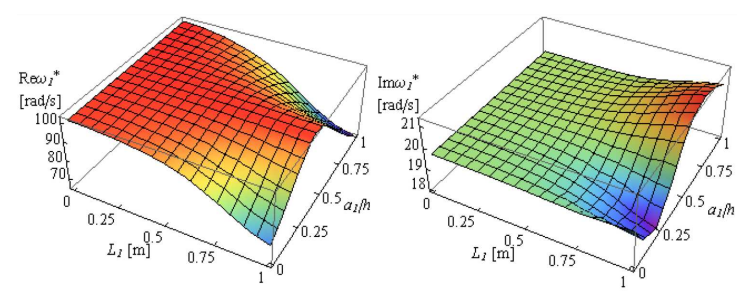

Fig. 4. The dependency of the first eigenvalue of frame on the first crack depth $a_{1} / h$ and its localization $L_{1}$.

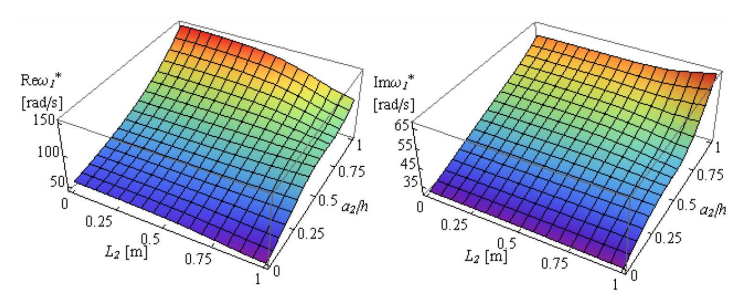

Fig. 5. The dependency of the first eigenvalue of frame on the second crack depth $a_{2} / h$ and its localization $L_{2}$.
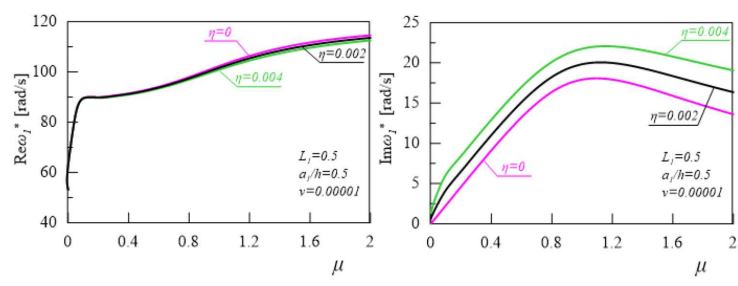

Fig. 6. The relationship between the first eigenvalue of frame and constructional damping $\mu$ for first crack.
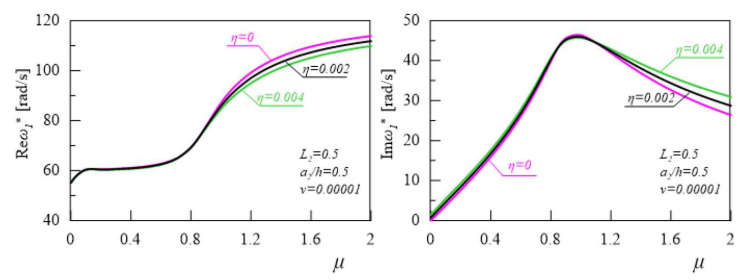

Fig. 7. The relationship between the first eigenvalue of frame and constructional damping $\mu$ for second crack. 
In Figs. 6 and 7, there are some areas in curves progression where the increase in the constructional damping $\mu$ leads to increase the values of the imaginary $\operatorname{Im}\left(\omega^{*}\right)$ part of eigenvalue to maximum.

\section{Conclusions}

Changes in the crack location cause significant changes in $\operatorname{Re}\left(\omega^{*}\right)$ and $\operatorname{Im}\left(\omega^{*}\right)$ of the first eigenvalue of the system. Some substantial changes also can be observed in the first eigenvalue of the system with changes in the crack depth.

Considerable changes can be also observed in the amplitude decay factor of first eigenvalue when the coefficient $\mu$ changes. An increase of constructional damping causes that the values of amplitude decay factor $\operatorname{Im}\left(\omega^{*}\right)$ increase to the highest values, followed by $\operatorname{Im}\left(\omega^{*}\right) \rightarrow 0$, where $\mu \rightarrow \infty$. Obtained results help to determine parameters for which the system is damped the most effective.

\section{References}

[1] W. Sochacki, M. Bold, J. Appl. Math. Comput. Mech. 15, 2 (2016).

[2] M. Bold, W. Sochacki, J. Vibroeng. 21, 1 (2019).

[3] M. Bold, W. Sochacki, J. Vibroeng. 20, 1 (2018).

[4] P.F. Rizos, N. Aspragathos, A.D. Dimarogonas, J. Sound Vib. 138, 3 (1990).

[5] S. Uzny, K. Sokół, J. Theor. Appl. Mech. 56, 1 (2018).

[6] J.W. Liang, B.F. Feeny, Nonlinear Dyn. 16, 337 (1998).

[7] Y.-R. Yang, J.-Y. Zhang, J. Sound Vib. 203, 795 (1997).

[8] M.I. Friswell, A.W. Lees, J. Sound Vib. 242, 2, (2001). 